\section{JTI}

JOURNAL OF

TRAUMA AND INJURY

Received: October 12, 2020

Accepted: December 3, 2020

\section{Correspondence to}

David G. Rabkin, M.D.

Department of Cardiothoracic Surgery, Loma Linda University Medical Center,

Coleman Pavilion, Suite 21121, 11175

Campus Street, Loma Linda, CA 92354

USA

Tel: +1-909-558-4354

Fax: +1-909-558-0348

E-mail:drabkin@llu.edu

\title{
Delayed Diagnosis of Traumatic Rupture of Anterior Papillary Muscle of Tricuspid Valve; Importance of Trans-Esophageal Echocardiogram in the Evaluation of Major Blunt Chest Trauma
}

Ryan Bylsma, M.D. ${ }^{1}$, Mustafa Baldawi, M.D. ${ }^{1}$, Bruce Toporoff, M.D. ${ }^{1}$, Matthew Shin, B.A. ${ }^{1}$, Meghan Cochran-Yu, M.D. ${ }^{2}$, Davinder Ramsingh, M.D. ${ }^{3}$, Purvi Parwani, M.B.B.S., M.P.H. ${ }^{4}$, David G. Rabkin, M.D. ${ }^{1}$

\footnotetext{
${ }^{1}$ Department of Cardiothoracic Surgery, Loma Linda University Medical Center, Loma Linda, CA, USA

${ }^{2}$ Division of Acute Care Surgery, Department of General Surgery, Loma Linda University Medical Center, Loma Linda, CA, USA

${ }^{3}$ Department of Anesthesia \& Critical Care, Loma Linda University Medical Center, Loma Linda, CA, USA

${ }^{4}$ Division of Cardiology, Department of Medicine, Loma Linda University Medical Center, Loma Linda, CA, USA
}

We present a case of delayed diagnosis of traumatic tricuspid valve rupture in a patient who was emergently brought to the operating room for repair of lacerations to the heart and liver without intraoperative transesophageal echocardiography (TEE). Initial postoperative transthoracic echocardiography (TTE) did not show structural pathology. One week later, TTE with better image quality showed severe tricuspid regurgitation. Subsequently, TEE clearly demonstrated rupture of the anterior papillary muscle and flail anterior tricuspid leaflet. The case description is followed by a brief discussion of the utility of TEE in the setting of blunt thoracic trauma.

Keywords: Blunt injury; Tricuspid regurgitation; Transesophageal echocardiography 


\section{INTRODUCTION}

Traumatic rupture of the tricuspid valve apparatus is a rare event, with about 100 cases reported in the literature. The diagnosis can be difficult to make due to a lack of acute physical findings and the presence of other traumatic injuries [1], and it is often made long after the causative injury [2]. Although the tricuspid valve is an anterior cardiac structure, transthoracic echocardiography (TTE) can be less effective than transesophageal echocardiography (TEE) in making the diagnosis in the setting of chest trauma [3]. We present the case of a 32-year-old man who suffered multiple injuries, including sternal fracture and cardiac laceration, during a high-speed motor vehicle accident. He was brought to the operating room directly from the emergency department for the repair of cardiac and liver lacerations. Neither TTE nor TEE was part of his initial or intraoperative evaluation. Focused TTE that was done the next morning ruled out cardiac tamponade as a source of hypotension, but failed to demonstrate structural cardiac pathology. Six days later, comprehensive TTE was performed to assess heart function in the setting of suspected septic shock. This time, TTE showed severe tricuspid regurgitation and dilatation of the right-sided cardiac chambers, thought to be due to tricuspid annular dilatation from elevated pulmonary pressure. Subsequent TEE clearly demonstrated avulsion of the anterior papillary muscle and flail anterior leaflet of the tricuspid valve with wide-open tricuspid insufficiency. Over the next 4 days, the patient's sepsis improved, but his renal and liver function began to deteriorate and he was brought to the operating room for an intervention on the tricuspid valve.

\section{CASE REPORT}

A previously fit 32-year-old male warehouse worker was brought to our emergency department as a level A trauma case after being an unrestrained driver of a large sport utility vehicle that crashed into the side of a building going approximately 40 miles per hour (or roughly 65 kilometers per hour), while the patient was inhaling keyboard cleaner and "blacking out." His pre-hospital
Glasgow Coma Scale score was 15. He was hypotensive on arrival and given two units of packed red blood cells with normalization of his blood pressure. On examination, he was found to have tenderness over his sternum as well as abdominal distention and tenderness. His laboratory values were unremarkable. His initial chest roentgenogram was notable only for fractures of the third to sixth right ribs and the second and fifth left ribs. Computed tomography (CT) showed contusion of the right upper and middle lung lobes, a left-sided pleural effusion suspicious for hemothorax, a mildly displaced comminuted posterior right acetabular fracture, and hypodensity of segment IV of the liver with perihepatic stranding suspicious for a laceration. A chest tube was placed on the left side and $1.5 \mathrm{~L}$ of blood was evacuated, prompting exploration.

The patient was brought directly to the operating room, where through an anterolateral thoracotomy, the pericardium was found to be widely ruptured. A partial-thickness laceration of the left ventricle was found and repaired with interrupted, pledgeted sutures. During this time the patient became hypotensive with an enlarging abdomen, and due to concerns regarding possible intra-abdominal hemorrhage he underwent exploratory celiotomy through a midline incision from xiphoid to the pubic symphysis. On examination of the liver, a grade IV laceration with active bleeding near segment IVb was noted. Bovie electrocautery, argon beam coagulation, and hepatorrhaphy with Zero-Chromic suture on a blunt needle were performed in an effort to achieve hemostasis. Although the bleeding improved, active hemorrhage continued. The abdomen was irrigated and packed with laparotomy pads, and an Abthera ${ }^{\mathrm{TM}}$ (3M Health Care, St. Paul, MN, USA) temporary abdominal closure device was applied. The patient was taken to the interventional radiology suite in critical condition for successful embolization of hepatic segments 3 and 4 with gel foam slurry and then to the trauma intensive care unit for resuscitation. His orthopedic injuries were initially treated with right lower extremity skeletal traction.

TTE was obtained the next morning due to hypotension to rule out cardiac tamponade (which it did); however, the image quality was suboptimal and tricuspid regurgitation was not detected. Over the next 6 days, the patient remained intubated with bilateral pulmonary 


\section{JTI}

contusions and poor gas exchange. TTE was performed again to evaluate his heart function with improved image quality and demonstrated severe tricuspid regurgitation with severe right ventricular dilatation (Fig. 1). This was attributed to elevated pulmonary pressure, but subsequent TEE showed rupture of the papillary muscle and a flail anterior tricuspid leaflet (Fig. 2A, C) with torrential tricuspid regurgitation (Fig. 2B) shunting right to left across a patent foramen ovale. At the time, the patient appeared to be septic and had very poor gas exchange. Over the course of the next several days after washout of his abdomen, his pressor requirements decreased considerably, gas exchange improved to the point where his fraction of inspired oxygen was 0.5 , and his liver and renal function began to deteriorate, suggesting that severe tricuspid regurgitation was having a significant impact on his renal
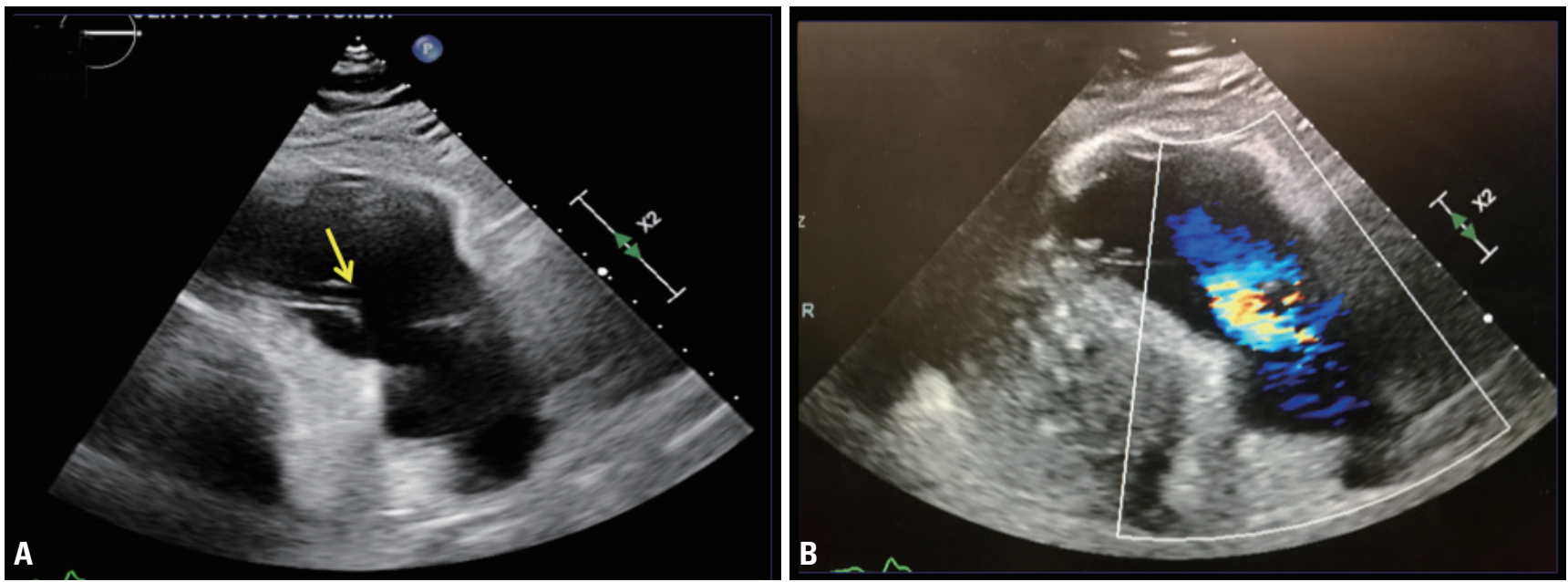

Fig. 1. Transthoracic echocardiogram taken on postoperative day 6 (A) without and (B) with a Doppler signal showing severe tricuspid regurgitation without a clear etiology. The arrow points towards the tricuspid valve.
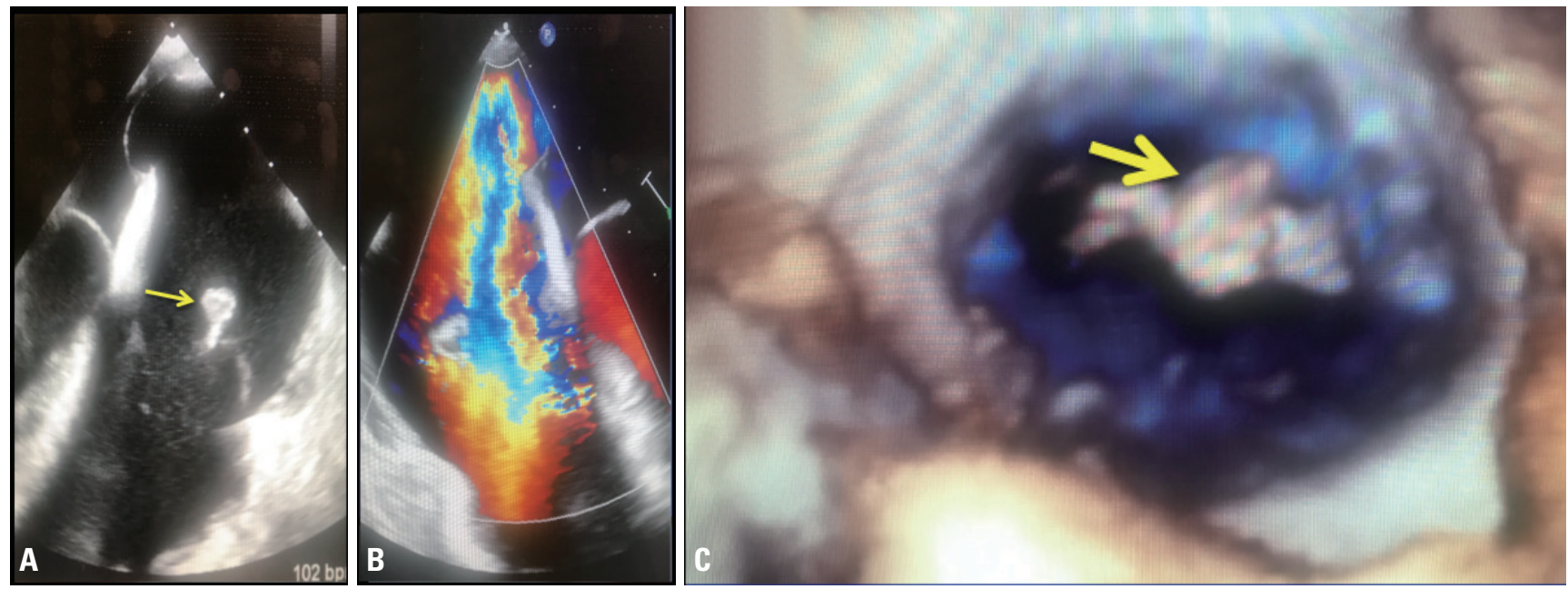

Fig. 2. Transesophageal echocardiogram taken on postoperative day 7 demonstrating a ruptured papillary muscle and flail anterior leaflet of the tricuspid valve. (A) Mid-esophageal long axis view. The arrow points to the ruptured papillary muscle still attached to the flail anterior leaflet. (B) Mid-esophageal 4-chamber view showing severe tricuspid regurgitation with a ruptured papillary muscle head and flail tricuspid valve. The inter-atrial septum appeared thinned out and bulged up with a tricuspid regurgitation jet. (C) Three-dimensional zoomed-in view showing the ruptured papillary muscle protruding into the right atrium (arrow). 
and hepatic physiology. We therefore took the patient back to the operating room 10 days after his index operation.

Through a median sternotomy, we exposed the tricuspid valve through an oblique incision in the right atrium. The anterior papillary muscle had ruptured and the anterior leaflet was untethered and flail (Fig. 3). There was also avulsion of the septal leaflet. We attempted repair by re-attaching the avulsed septal leaflet with a running 5-0 monofilament suture and used another 5-0 pledgeted monofilament suture to re-attach the papillary muscle to the right ventricle. Insufflation of the right ventricle with saline appeared to demonstrate competency of the valve and we placed a \#36 annuloplasty ring. We also repaired a large, patent foramen ovale. Upon waning from cardiopulmonary bypass, there was at least moderate tricuspid valve regurgitation; therefore, we resumed full cardiopulmonary bypass, removed the annuloplasty ring and replaced the valve with a $\# 27$ bioprosthesis. We then weaned the patient from bypass uneventfully in a normal sinus rhythm.

After multiple washouts, his abdomen was closed with retention sutures and he was discharged to a rehabilitation facility after a 35-day hospital stay. He was discharged

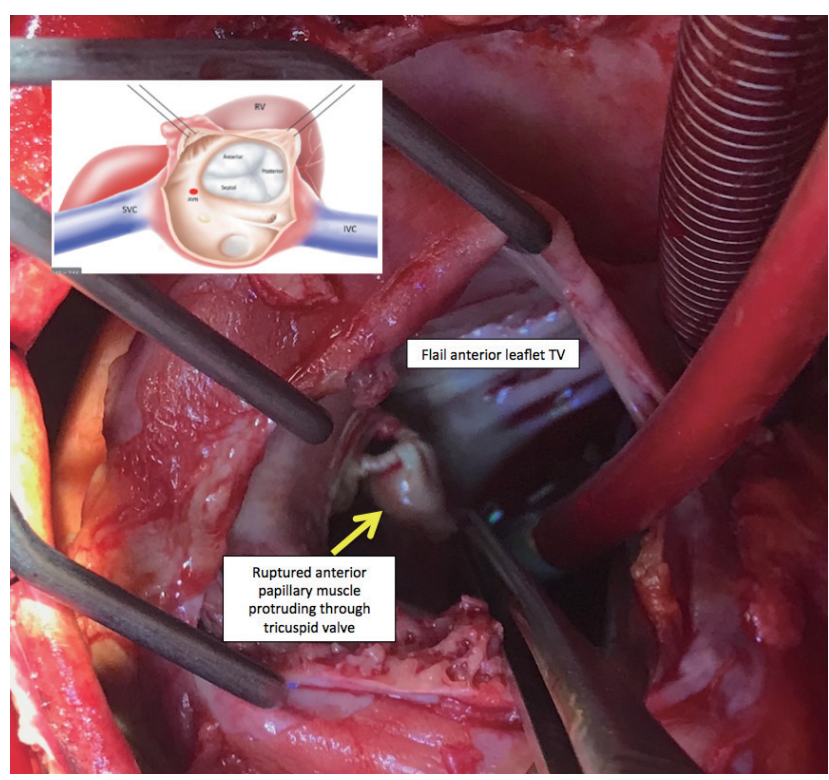

Fig. 3. Intraoperative image of the ruptured papillary muscle projecting into the right atrium through the flail anterior leaflet of the tricuspid valve. home 2 weeks later. At a follow-up visit 3 months later, he had made a full recovery from his thoracic and abdominal injuries, but remained non-weight-bearing on the right lower extremity.

\section{DISCUSSION}

Trauma is the leading cause of death in people younger than 45 years old in the United States. Motor vehicle collisions represent the most common cause of major thoracic injury among patients presenting to emergency departments, and blunt chest trauma is a major source of morbidity and mortality both domestically and globally [4]. Ventricular contusion is the most common cardiac injury after major blunt chest trauma, but free wall rupture, aortic injury, septal rupture and valvular disruption have all been previously described $[5,6]$. Our case illustrates the difficulty in using TTE in the setting of blunt thoracic trauma where the acoustic window may be obscured by inflamed tissue planes, hematoma, chest tubes, and bandages. The initial TTE did not reveal what was later described as "torrential" tricuspid regurgitation, and subsequent TTE was not able to demonstrate the mechanism that was clearly shown on TEE. While traumatic rupture of the tricuspid valve is rarely reported, the diagnosis is often delayed for years. A classic series of 13 cases reported a median interval between injury and operative intervention of 17 years [2], and the true incidence is likely underreported [1]. Prompt diagnosis is useful, even if the patient is asymptomatic, since repair strategies are more often successful prior to the development of irreversible right ventricular dysfunction [2].

Initial resuscitation and management of trauma patients is based on Advanced Trauma Life Support protocols, which in the setting of blunt chest trauma emphasize focused assessment with sonography for trauma [7], an excellent method of demonstrating pericardial or pleural effusion or pneumothoraces, but one that is suboptimal for diagnosing structural heart problems [8]. CT is also emphasized in protocols for patients with blunt chest trauma, but it is similarly inadequate for diagnosing structural heart pathology and is contraindicated in patients who need emergent exploration. In the acute setting, these 
diagnostic modalities are useful given the widespread availability of the technology and the dissemination of the knowledge required to perform and interpret the studies. TEE is less widely available in the acute setting, but is increasingly available in trauma center operating rooms [9].

Although given this patient's intra-abdominal hemorrhage, it is unlikely that we would have chosen to address the tricuspid valve pathology in the index operation, awareness of severe tricuspid regurgitation may have facilitated his subsequent management. Regardless, the case illustrates the importance of TEE in the setting of blunt thoracic trauma. Indeed, the utility of perioperative TEE for the management of trauma patients has been supported [10]. Various trauma severity indices including the thoracic component of the New Injury Severity Score [11] and the Thorax Trauma Severity Score [12] can help stratify blunt chest trauma into major and non-major.

In the setting of major blunt force trauma to the chest with or without apparent external cardiac injury, intraoperative TEE is an indispensable adjunct to rule out concomitant structural injuries, and we suggest that it should be used routinely. In patients with major blunt chest trauma who do not have indications for an operative intervention, TEE may neither be practical or necessary in the acute setting, but we feel that it should be a routine part of patients' evaluation prior to discharge.

\section{REFERENCES}

1. Gayet C, Pierre B, Delahaye JP, Champsaur G, Andre-Fouet X, Rueff $\mathrm{P}$. Traumatic tricuspid insufficiency. An underdiagnosed disease. Chest 1987;92:429-32.

2. van Son JA, Danielson GK, Schaff HV, Miller FA Jr. Traumatic tricuspid valve insufficiency. Experience in thirteen patients. J Thorac Cardiovasc Surg 1994;108:893-8.
3. Nelson M, Wells G. A case of traumatic tricuspid valve regurgitation caused by blunt chest trauma. J Am Soc Echocardiogr 2007;20:198.e4-5.

4. Veysi VT, Nikolaou VS, Paliobeis C, Efstathopoulos N, Giannoudis PV. Prevalence of chest trauma, associated injuries and mortality: a level I trauma centre experience. Int Orthop 2009;33:1425-33.

5. Banning AP, Durrani A, Pillai R. Rupture of the atrial septum and tricuspid valve after blunt chest trauma. Ann Thorac Surg 1997;64:240-2.

6. Kulshrestha P, Das B, Iyer KS, Sampath KA, Sharma ML, Rao IM, et al. Cardiac injuries--a clinical and autopsy profile. J Trauma 1990;30:203-7.

7. Committee on Trauma. ATLS advanced trauma life support 10th edition student course manual. Chicago:American College of Surgeons;2018.

8. Baker L, Almadani A, Ball CG. False negative pericardial focused assessment with sonography for trauma examination following cardiac rupture from blunt thoracic trauma: a case report. J Med Case Rep 2015;9:155.

9. Leichtle SW, Singleton A, Singh M, Griffee MJ, Tobin JM. Transesophageal echocardiography in the evaluation of the trauma patient: a trauma resuscitation transesophageal echocardiography exam. J Crit Care 2017;40:202-6.

10. Griffee MJ, Singleton A, Zimmerman JM, Morgan DE, Nirula $\mathrm{R}$. The effect of perioperative rescue transesophageal echocardiography on the management of trauma patients. A A Case Rep 2016;6:387-90.

11. Osler T, Baker SP, Long W. A modification of the injury severity score that both improves accuracy and simplifies scoring. J Trauma 1997;43:922-5.

12. Aukema TS, Beenen LF, Hietbrink F, Leenen LP. Validation of the thorax trauma severity score for mortality and its value for the development of acute respiratory distress syndrome. Open Access Emerg Med. 2011;3:49-53. 